\title{
Decadal correlation between crustal deformation and variation in length of day of the Earth
}

\author{
Qing-Liang Wang ${ }^{1 *}$, Yun-Tai Chen ${ }^{2}$, Du-Xin Cui ${ }^{1}$, Wen-Ping Wang ${ }^{1}$, and Wei-Feng Liang ${ }^{1}$ \\ ${ }^{1}$ Second Center for Crustal Deformation Monitoring, CSB, Xian 710054, China \\ ${ }^{2}$ Institute of Geophysics, CSB, Beijing 100081, China
}

(Received December 31, 1999; Revised September 2, 2000; Accepted September 19, 2000)

\begin{abstract}
Recently, in comparing with Earth rotation data, we found some exciting correlation phenomena between lengthof-day (LOD) and crustal deformations as well as stress observations in China, the correlation appears most typically on decade time scales and seems to be direction-dependent. As LOD variation induced stress and deformation is far less than the observed ones, we infer that decade variations in crustal deformation and stress are very possibly attributed to the core-mantle coupling process, just like LOD decade variations are attributed to core-mantle coupling. More detailed and extended investigations are going on, and we believe that the similar correlation phenomena should also exist in other part of the world. The LOD-correlated crustal deformations are expected to provide a better constraint on verifying the real core-mantle coupling mechanism, topographic, electromagnetic or gravitational coupling.
\end{abstract}

\section{Introduction}

Astronomical observations demonstrate that the Earth does not spin at a stable rate. The variations in rotation rate or length of day (LOD) can be roughly classified into three types: secular deceleration, periodic variations and irregular undulations. The secular deceleration, about 1-2 ms increase in length of day per century, is due primarily to friction of ocean tides on the surface of the Earth (Lambeck, 1980). Periodic LOD fluctuations, typically on annual and semi-annual time scales, are mainly excited by global atmospheric circulation and solar tide (Lambeck, 1980). The reason for irregular LOD fluctuations has not been well determined, but variations on decade time scales, are generally attributed to exchanges of angular momentum between the core and the mantle. For example, by inferring the core surface flow from geomagnetic models, Jault et al. (1988), Jault and Le mouel (1990) have shown that changes in the length of day and corresponding changes in the angular momentum of the mantle are roughly correlated with changes in the angular momentum of the core, and very recently, Pais and Hulot (2000) further showed an agreement between the observed LOD decade change and the core surface flow-based prediction of Jault et al. (1988).

To be noticed, the observed LOD variations are actually the changes in rotation rate of the mantle as a global body. From the core-mantle coupling mechanisms for LOD decade variation, we are naturally interested in whether the core-mantle coupling can modulate the mantle or crustal deformation on

\footnotetext{
*Now at Institute of Geophysics, China Seismological Bureau, as a doctoral student.

Copy right (c) The Society of Geomagnetism and Earth, Planetary and Space Sciences (SGEPSS); The Seismological Society of Japan; The Volcanological Society of Japan; The Geodetic Society of Japan; The Japanese Society for Planetary Sciences.
}

decade time scales. In our recent investigations, we do have found some affirmative evidences from the decade correlation between LOD and crustal deformations. In present paper, we will firstly report the new correlation finding, and then discuss its implication to geodynamics.

\section{Decade Correlation between LOD and Crustal Deformations}

In order to detect the possible earthquake precursors, there have been nearly 300 fault deformation monitoring sites, over 100 tilt gauge stations and stress observation stations established in China by China Seismological Bureau (CSB) since early $1970 \mathrm{~s}$. Where the fault deformation sites are regularly monitored once to 12 times per year by leveling, baseline measurement and laser ranging, the tilt and stress gauges are regularly observed at one-day interval.

In early 1999 , in comparing the time series of fault deformations with Earth orientation parameters (EOP) downloaded from IERS, we surprisingly found a lot of correlation phenomena between LOD and fault deformations. Later on, in the tilt and stress observations in China, we also found some similar correlation with LOD. The LOD-correlated fault deformation is generally several millimeters, the LODcorrelated tilt change is generally several arc seconds, and the maximum LOD-correlated stress variation can reach several hundred $\mathrm{KPa}$. The statistic results of correlation coefficients to LOD are listed in Table 1, where the mean correlation coefficients of fault deformations, tilt observations and stress/strain observations are around 0.6.

Figures 1 to 3 present some representative examples of the correlation, the corresponding site illustration and correlation coefficients are listed in Table 2. To be mentioned, the LOD variations in Figs. 1 through 3 are based on IERS data, and the components with period less than one year have been 
Table 1. Statistics of correlation coefficents to LOD variation.

\begin{tabular}{cccccc}
\hline $\begin{array}{c}\text { Observation } \\
\text { Data }\end{array}$ & $\begin{array}{c}\text { Minimum } \\
\text { coefficient }\end{array}$ & $\begin{array}{c}\text { Maximum } \\
\text { coefficient }\end{array}$ & $\begin{array}{c}\text { Mean } \\
\text { coefficient }\end{array}$ & $\begin{array}{c}\text { Standard } \\
\text { deviation }\end{array}$ & $\begin{array}{c}\text { Size of } \\
\text { samples }\end{array}$ \\
\hline Fault deformation & 0.10 & 0.97 & 0.61 & 0.21 & 339 \\
Tilt observation & 0.13 & 0.93 & 0.59 & 0.22 & 53 \\
Stress/strain observation & 0.13 & 0.88 & 0.59 & 0.19 & 56 \\
\hline
\end{tabular}

Table 2. Representative monitoring stations with observations correlated to LOD variation.

\begin{tabular}{ccccccc}
\hline Stations & Abbreviation & Station property & Latitude & Longitude & Component & Correlation coefficient \\
\hline Beipiao & $\mathrm{BP}$ & Fault deformation & $41.77^{\circ} \mathrm{N}$ & $120.78^{\circ} \mathrm{E}$ & Cross-fault & 0.86 \\
Tangshan & $\mathrm{TS}$ & Fault deformation & $39.50^{\circ} \mathrm{N}$ & $118.28^{\circ} \mathrm{E}$ & Cross-fault & 0.68 \\
Bashagou & $\mathrm{BSG}$ & Fault deformation & $34.08^{\circ} \mathrm{N}$ & $104.38^{\circ} \mathrm{E}$ & Cross-fault & 0.76 \\
Anguoozhen & $\mathrm{AGZ}$ & Fault deformation & $35.63^{\circ} \mathrm{N}$ & $106.48^{\circ} \mathrm{E}$ & Cross-fault & 0.71 \\
Yinchuan & $\mathrm{YC}$ & Tilt observation & $38.50^{\circ} \mathrm{N}$ & $106.15^{\circ} \mathrm{E}$ & $\mathrm{SN}$ & 0.68 \\
Chuxion & $\mathrm{CX}$ & Tilt observation & $25.02^{\circ} \mathrm{N}$ & $101.42^{\circ} \mathrm{E}$ & $\mathrm{SN}$ & 0.72 \\
Huhehaote & $\mathrm{HH}$ & Tilt observation & $40.62^{\circ} \mathrm{N}$ & $111.53^{\circ} \mathrm{E}$ & $\mathrm{EW}$ & 0.90 \\
Xibozi & $\mathrm{XB}$ & Tilt observation & $40.40^{\circ} \mathrm{N}$ & $116.00^{\circ} \mathrm{E}$ & $\mathrm{EW}$ & 0.92 \\
Yongming & $\mathrm{YN}$ & Stress observation & $22.85^{\circ} \mathrm{N}$ & $108.28^{\circ} \mathrm{E}$ & $\mathrm{EW}$ & 0.67 \\
Songming & $\mathrm{SM}$ & Stress observation & $25.42^{\circ} \mathrm{N}$ & $103.20^{\circ} \mathrm{E}$ & $\mathrm{EW}$ & 0.67 \\
Wenxian & $\mathrm{WX}$ & Stress observation & $33.00^{\circ} \mathrm{N}$ & $104.65^{\circ} \mathrm{E}$ & $\mathrm{NE}$ & 0.69 \\
Jingning & $\mathrm{JN}$ & Stress observation & $35.50^{\circ} \mathrm{N}$ & $105.60^{\circ} \mathrm{E}$ & $\mathrm{NS}$ & 0.73 \\
\hline
\end{tabular}

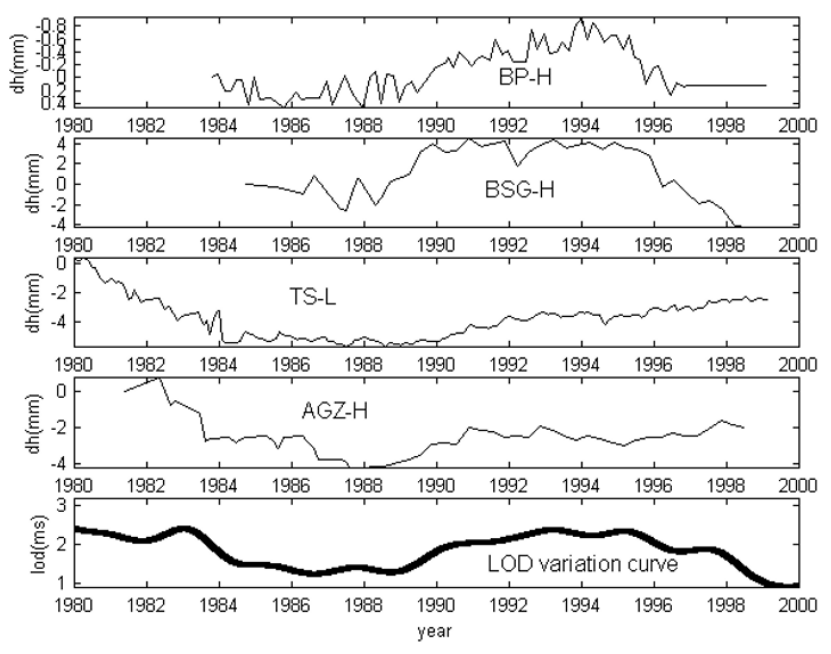

Fig. 1. Representative correlation between LOD and fault deformations of site BP, BSG, TS and AGZ. The $\mathrm{H}$ and $\mathrm{L}$ after the abbreviated site name indicate respectively the vertical deformation and horizontal deformation.

eliminated by filtering. The plots of the tilt observations in Fig. 2 are based on five-day values, and those of stress observations in Fig. 3 are based on one-day values.

As investigated, the correlation between LOD and crustal deformations mainly shows the following three characteris-
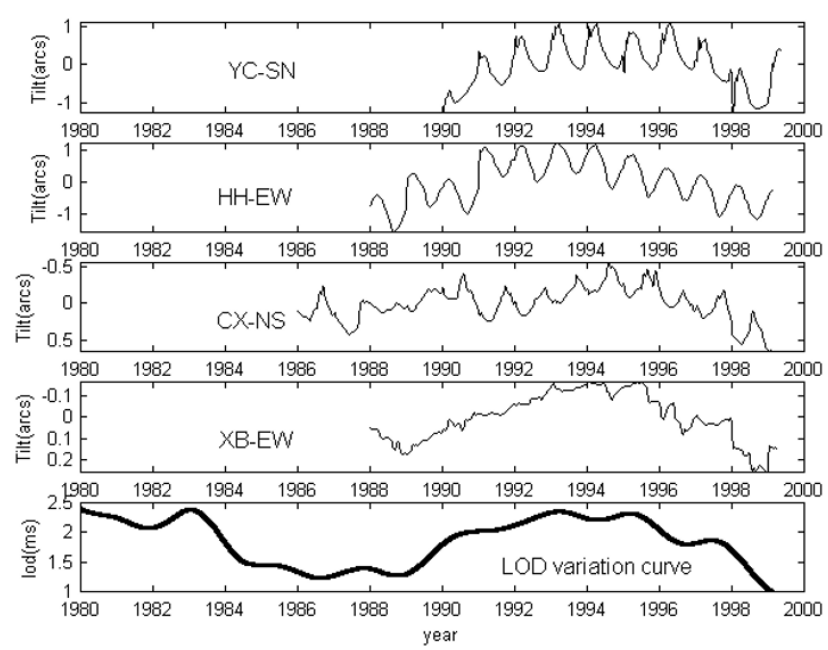

Fig. 2. Representative correlation between LOD and tilt observations of station YC, HH, CX and XB. The linear trend of the top three have been removed in order to eliminate drift, EW and NS here indicate the direction of the tilt component.

tics. (i) The correlation appears most typically on decadal time scales, and for some tilt observations, annual tilt-LOD correlation can also be recognized clearly. (ii) The correlation is direction dependent, Fig. 4 is a representative example of the direction dependence of correlation at the Xibozi tilt 


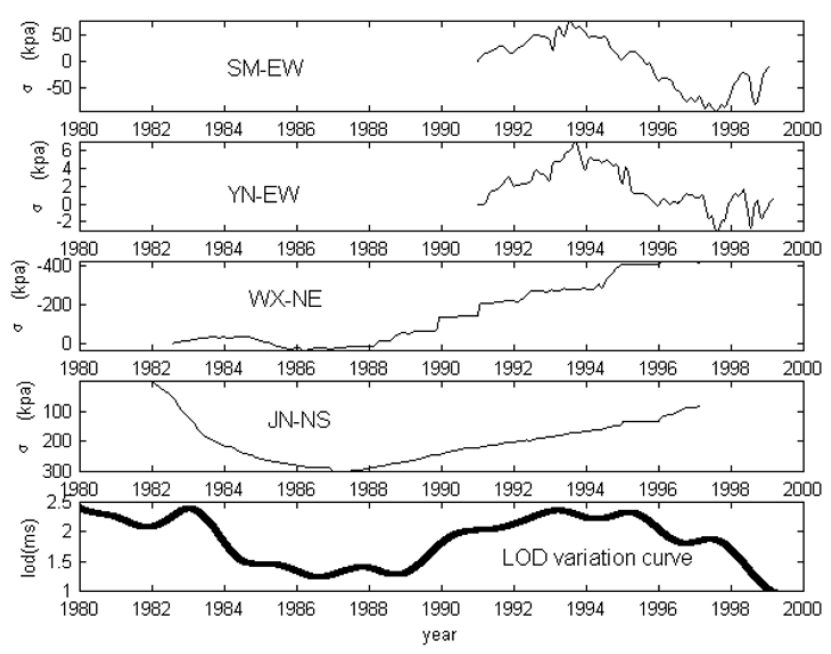

Fig. 3. Representative correlation between LOD and stress observations of station SM, YN, WX and JN. The EW, NE and NS after the abbreviated station name indicate direction.
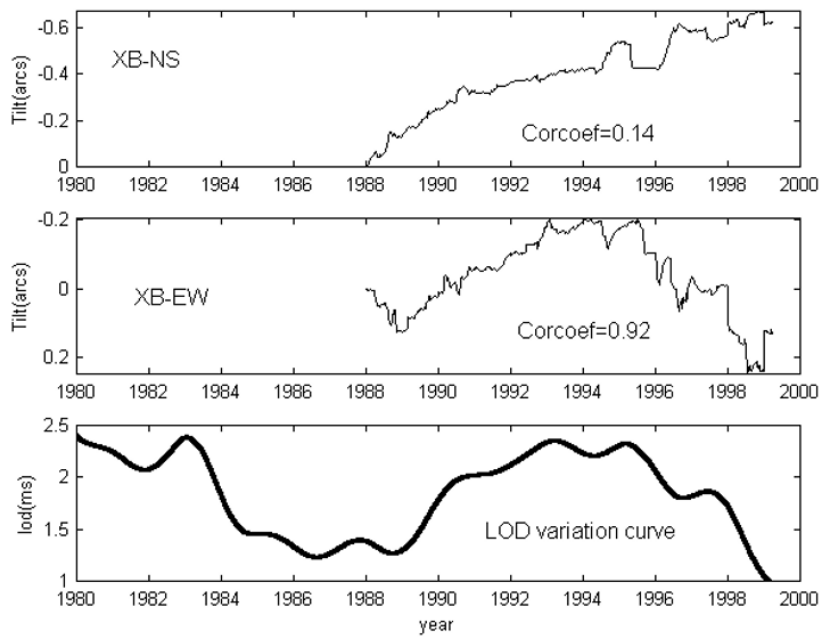

Fig. 4. Representative correlation of XB tilt station. Where the correlation of the EW component to LOD is much better than that of the NS component.

gauge station near Beijing, China, where the correlation of EW component to LOD is much better than that of the NS component. (iii) The correlation is tectonic structure dependent, for example, at the same fault deformation monitoring site, the correlation of the cross-fault deformation to LOD is generally better than those within the same block of the fault.

\section{Mechanisms for Decade Correlation}

In principle, there are only two possible mechanisms responsible for the decade correlation between LOD variation and crustal deformation as well as stress: First, decade variations in crustal deformation and stress are induced by variation in Earth's rotation rate or LOD. Second, the correlated decade variations in LOD, crustal deformation and stress all originate from a same dynamic process within or outside the earth.

Wahr (1985) and Gipson et al. (1998) have theoretically derived the site displacement induced by LOD variation and pole motion. In present paper, we follow their study to examine the possibility of the first mechanism. In their work, the site displacement caused by LOD variation can be expressed as:

$$
\begin{aligned}
& \Delta U=h m_{z} \frac{\Omega^{2} r^{2}}{g} \sin ^{2} \theta \\
& \Delta E=0 \\
& \Delta N=l m_{z} \frac{\Omega^{2} r^{2}}{g} \sin 2 \theta .
\end{aligned}
$$

Where $\Delta U, \Delta E$ and $\Delta N$ are respectively the vertical, east and north displacements caused by LOD variation, $\Omega$ is the average angular velocity of the Earth, $\theta$ is the colatitude, $r$ is the radius from geocenter, $g$ is the gravitational acceleration at the Earth's surface, $m_{z}$ is the excess length of day (LOD), $h$ and $l$ are respectively the vertical and horizontal Love number, $h \approx 0.60$ and $l \approx 0.085$.

From (1) to (3), the tilt and strain changes of the eastwest, north-south and vertical components induced by LOD variation can be derived as

$$
\begin{aligned}
\Delta T_{e w} & =\frac{\partial(\Delta U)}{\partial(r \lambda \sin \theta)}=0 \\
\Delta T_{n s} & =\frac{\partial(\Delta U)}{\partial(r \theta)}=-h \frac{\Omega^{2} r}{g} m_{z} \sin 2 \theta \\
\Delta \varepsilon_{e w} & =\frac{\partial(\Delta E)}{\partial(r \lambda \sin \theta)}=0 \\
\Delta \varepsilon_{n s} & =\frac{\partial(\Delta N)}{\partial(r \theta)}=2 l m_{z} \frac{\Omega^{2} r}{g} \cos 2 \theta \\
\Delta \varepsilon_{v v} & =\frac{\partial(\Delta U)}{\partial r}=-2 h m_{z} \frac{\Omega^{2} r}{g} \sin ^{2} \theta .
\end{aligned}
$$

Where $\lambda$ is the longitude, $\Delta T_{e w}$ and $\Delta T_{n s}$ indicate the eastwest and north-south tilt changes induced by LOD variation, $\Delta \varepsilon_{e w}, \Delta \varepsilon_{n s}$ and $\Delta \varepsilon_{v v}$ are respectively the east-west, northsouth and vertical strain changes.

Substituting the nominal values of $\Omega, g$ and $r$ into (1) through (8), and setting the maximum excess length of day $m_{z}=2.5 \mathrm{~ms} /$ day during the year 1980 through 2000, we can estimate that the maximum tilt change induced by LOD variation is of the order of $\sim 10^{-4}$ arc second. And if setting the elastic modulus of the crust rock being of the order of $\sim 10^{10} \mathrm{~Pa}$, we can further estimate that the maximum principle stress change caused by LOD variation is only of the order of $\sim 10^{-1} \mathrm{~Pa}$.

From the above theoretical study we can see that, there are several key contradictions in applying the first mechanism to account for the decade correlation between LOD variation and crustal deformation. (i) LOD variation could not induce tilt or strain/stress change in east-west direction, but the actually observed tilt and stress do often correlate well with LOD on decade time scales. (ii) The observed LODcorrelated tilt change is often of the order of $10^{-1}$ to 100 arc second, the maximum LOD-induced tilt change is only of order $\sim 10^{-4}$, the later is a factor of $10^{-4} \sim 10^{-3}$ smaller than the former. (iii) The observed LOD-correlated stress change is of the order of $10^{4} \sim 10^{5} \mathrm{~Pa}$, the theoretical LOD-induced stress change is only of the order of $\sim 10^{-1} \mathrm{~Pa}$, which is a factor of $10^{-5} \sim 10^{-6}$ smaller than the former. 
With the first mechanism invalid, we infer that the correlated decade variations in LOD, crustal deformation and stress all originate from the same source, and the source itself, as investigated on decade LOD variation, is most likely the core-mantle coupling process deep in the Earth.

\section{Concluding Remarks}

In this paper, we have presented some exciting decade correlation phenomena between LOD variation and crustal deformation as well stress observations in China. We believe the similar correlation phenomenon should have also existed in other part of the world and in other geophysical observation data. Very recently, we do have found some similar LOD-tilt/strain decade correlation in Italy and in the geotemperature records of China. In order to examine the global behavior of the decade correlation, a wide international cooperation and data exchange is necessary. To be noticed that, due to the complexity of contributing force to crustal movement, we should not expect the decade LOD-deformation correlation always appear on all monitoring sites or stations.

We have theoretically demonstrated that decade LOD variation is not able to cause the observed correlated changes in tilt and stress, and we have attributed the LOD-correlated decade changes in crustal deformation and stress to coremantle coupling mechanism. Keeping this mechanism in mind, we infer that, core-mantle coupling is actually an important driving force of crustal deformation, in other word, core-mantle coupling contributes a lot to temporal undulation of crustal movement.

On core-mantle coupling, many coupling mechanisms have been proposed, among these, topography coupling (Hide, 1969, 1995; Hinderer et al., 1990), electromagnetic coupling (Rochester, 1968; Holm, 1998) and gravitational coupling (Buffett, 1996, 1999) are three possible candidates for LOD decade variation. But due to the lack of constraint, none of the core-mantle coupling mechanisms has been properly verified. We expect the LOD-correlated crustal deformation and stress observations may provide a reasonable constraint in determining the real core-mantle coupling. In order to do so, the theoretical models describing how the crust or lithosphere responses to the core-mantle coupling are urgent.

Acknowledgments. We thank Ms. Jiao Qing and Mr. Yi Zhi-Gang of Institute of Crust Stress, CSB, for providing some stress and fault deformation observation data of China, we thank Dr. Niu An-Fu of Wuhan Seismological Institute, CSB, for preparing the tilt observation data of China for us, we also thank Prof. Dennis D. McCarthy of U.S. Naval Observatory, for providing the LOD data before 1962. This research has been supported by the Basic Research Project G1998040703 of China and Research Project 95-09-01 of CSB.

\section{References}

Buffet, B. A., A mechanism for decadal fluctuations in the length of day, Geophys. Res. Lett., 23, 3803-3806, 1996.

Buffett, B. A., Free oscillations in the length of day: Inferences on physical properties near the core-mantle boundary, The Core-Mantle Boundary Region, Geodynamics Series, 28, 153-166, 1999.

Gipson, J. M. and C. P. Ma, Site displacement due to variation in Earth rotation, J. Geophys. Res., 103, 7337-7350, 1998.

Hide, R., Interaction between the Earth's liquid core and solid mantle, $\mathrm{Na}$ ture, 222, 1055-1056, 1969.

Hide, R., The topographic torque on a bounding surface of a rotating gravitating fluid and the excitation by core motions of decadal fluctuations in the Earth's rotation, G.J.R., 22,961-964, 1995.

Hinderer, J., H. Legros, D. Jault, and J. Le Mouel, Core-mantle topographic torque: a spherical harmonic approach and implications for the excitation of the Earth's rotation by core motions, Phys. Earth Planet. Inter., 59, 329-341, 1990.

Holm, R., Electromagnetic core-mantle coupling I, Explaining decadal changes in length of day, Geophys. J. Int., 132, 167-180, 1998.

Jault, D. and J. Le Mouel, Core-mantle boundary shape: constraints inferred from the pressure torque acting between the core and mantle, Geophys. J. Int., 101, 233-241, 1990.

Jault, D., C. Gire, and J. J. Le Mouel, Westward drift, core motions and exchanges of angular momentum between core and mantle, Nature, 333, 353-356, 1988.

Lambeck, K., The Earth's Variable Rotation, 397 pp., Cambridge Univ. Press, New York, 1980.

Pais, A. and G. Hulot, Length of day decade variations, torsional oscillations and inner core superrotation: evidence from recovered core surface zonal flows, Phys. Earth Planet. Int., 118, 291-316, 2000.

Rochester, M. G., Perturbations in the Earth's rotation and geomagnetic core-mantle coupling, J. Geomag. Geoelectr., 20, 387-402, 1968.

Wahr, J. M., Deformation induced by pole motion, J. Geophys. Res., 90, 9363-9368, 1985.

Q.-L. Wang (e-mail: wangql@public.xa.sn.cn), Y.-T. Chen (e-mail: chenyt@public.bta.net.cn), D.-X. Cui, W.-P. Wang, and W.-F. Liang 